\title{
Food Science, Foodomics and Capillary Electromigration Methods
}

Nowadays, boundaries between the different research disciplines are becoming more and more diffuse giving rise to impressive possibilities in the emerging interdisciplinary areas. In food science and nutrition, this trend has given rise to the development of new methodologies in which advanced analytical methodologies are applied to investigate topics considered unapproachable a few years ago. As a result, researchers in food science and nutrition are being pushed to move from classical methodologies to more advanced strategies, usually borrowing methods well established in medical, pharmacological and/or biotechnology research. This trend has generated the emergence of new areas of research, which usually try to dissect the research problem into smaller and more feasible challenges and with them a completely new terminology. Thus, terms such as foodomics, nutritional genomics, transgenics, functional foods, nutraceuticals, genetically modified (GM) foods, etc., are nowadays frequently used in food science. In this frame, the use of advanced analytical methodologies (including capillary electromigration methods) for food analysis is becoming a very important topic in which research institutions, agencies, regulatory laboratories and scientific instrumentation manufacturers are combining efforts to acquire the needed knowledge on food composition, quality, safety and/or biological activity.

The interest of the scientific community in food analysis and the different trends in this area are well documented by the present special issue on the use of advanced electromigration methods in "Food and Beverage Analysis". Namely, this issue presents six review papers, five of them are focused on the use of capillary electromigration techniques for detecting enantiomers, nucleosides, nucleotides, pesticides and contaminants in foods as well as foodborne pathogens. The sixth review deals with the application of 2-D electrophoresis to investigate food allergens.

A series of original contributions are also presented in this issue, describing the use of $\mathrm{CE}, \mathrm{CEC}$ and MEKC techniques to analyze proteins, phenolic compounds, heterocyclic and biogenic amines, mycotoxins, hippuric acid, carnitine, cyromazine, melamine and melatonin in foods. Moreover, works on the detection of genetically modified foods by CGE and the analysis of antibiotics and antioxidants by microemulsion electrokinetic chromatography are also presented. Interestingly, CEMS is becoming a common technique in food analysis laboratories as can be concluded from the growing number of contributions on this topic. As a good example, works on the use of CE-MS to analyze endocrine disruptors and polyphenols in different food matrices are presented in this special issue, plus a work on the speciation analysis of arsenic and selenium compounds in different foodstuffs by capillary electrophoresis-inductively coupled plasma-mass spectrometry. As editor of this special issue devoted to "Food and Beverage Analysis", I would like to thank all the authors for their suitable contributions, all reviewers for the time they devoted to the evaluation of the papers, Prof. Ziad El Rassi for his help and support, and to those of Electrophoresis team who contributed with their effort for the preparation of this special issue. 